\title{
SOME GEOMETRICAL PROPERTIES OF INFINITE-DIMENSIONAL BILINEAR CONTROLLED SYSTEMS
}

\author{
NACEURDINE BENSALEM and FERNAND PELLETIER \\ Université de Savoie \\ LAMA Campus Scientifique \\ 73376 Le Bourget du Lac cedex, France \\ E-mail:bensalem@univ-savoie.fr, pelletier@univ-savoie.fr
}

1. Introduction. The study of controlled infinite-dimensional systems gives rise to many papers (see for instance $[\mathrm{GXL}],[\mathrm{GXB}],[\mathrm{X}]$ ) but it is also motivated by various mathematical problems: partial differential equations ([BP]), sub-Riemannian geometry on infinite-dimensional manifolds ([Gr]), deformations in loop-spaces ([AP], [PS]). The first difference between finite and infinite-dimensional cases is that solutions in general do not exist (even locally) for every given control function. The aim of this paper is to study "infinite bilinear systems" on Hilbert spaces for which such a solution always exists. Moreover, to this particular class of controlled systems a nilpotent Lie algebra of degree 2 is naturally associated. On the other hand, given a Hilbertian nilpotent Lie algebra $\mathcal{G}$ of degree 2 we can associate to it in a natural way a bilinear system corresponding to left invariant distributions on a connected Lie groups $G$ whose Lie algebra is $\mathcal{G}$. The first result we obtain is an accessibility one which can be considered as a version of Chow's theorem in this situation. If we consider infinite-dimensional time optimal controlled systems the optimal trajectories are always abnormal curves which can be defined as in the finite-dimensional case. The second result of this paper is to give a "localization" of such curves: each of them is actually "normal" in some induced system on a submanifold. Finally we illustrate these results in the case of classical infinite generalized Heisenberg algebras.

\section{Preliminaries}

2.1. Infinite-dimensional controlled systems. Let $E$ be a separable Hilbert space in which we choose an orthonormal basis $\left\{e_{\lambda}: \lambda \in \mathbb{N}\right\}, F$ a Hilbertian subspace of $E$ and

1991 Mathematics Subject Classification: 22, 34, 47, 53, 93.

The paper is in final form and no version of it will be published elsewhere. 
$\left\{X_{i}: i \in \mathbb{N}\right\}$ a family of smooth vector fields on $E$ such that at each point $x$ in $E$ it is an orthonormal basis for $F$.

For every $x$ in $E$ we have a decomposition

$$
X_{i}(x)=\sum_{\lambda} X_{i}^{\lambda}(x) e_{\lambda} \quad(i \in \mathbb{N}, \lambda \in \mathbb{N})
$$

where $X_{i}^{\lambda}: E \rightarrow \mathbb{R}$ are smooth functions.

Let $\mathbb{U}=\left\{\left(u^{i}\right)\right\}$ be the space of absolute square convergent real series, i.e. $\mathbb{U}=\ell^{2}(\mathbb{N})$. Consider an open subinterval $J$ of $\mathbb{R}$ which contains 0 and a real number $c>0$ such that $J_{c}=[-c,+c] \subset J$. Given a map $u: J \rightarrow \mathbb{U}$ such that for every $0<b \leq c$ we have $u \in L^{2}\left(J_{b}, \mathbb{U}\right)$. Finally, consider the dynamical system

$$
\dot{x}=\sum_{\lambda} \sum_{i} u^{i}(t) X_{i}^{\lambda}(x) e_{\lambda}=\sum_{i} u_{i} X_{i} .
$$

Such a system possesses solutions under additional conditions given for instance in the following quite classical theorem (for a proof see for instance [Be]):

Theorem 2.1. Consider an open subinterval $J$ of $\mathbb{R}$ which contains 0 . Let $V$ be an open set of a separable Hilbert space $E$. Let $x_{0}$ be a point in $V$ and $a \in(0,1)$ a real number such that the closed ball centered at $x_{0}$ with radius $3 a, \overline{B\left(x_{0}, 3 a\right)}$ is contained in $V$.

Then we can find a real number $b>0$ such that for every $x$ in $\overline{B\left(x_{0}, a\right)}$ there exists a unique flow

$$
\alpha: J_{b} \times \overline{B\left(x_{0}, a\right)} \rightarrow V
$$

such that each curve

$$
\alpha(\cdot, x): J_{b} \rightarrow V
$$

is a solution of $(\mathrm{D})$ with $\alpha(0, x)=x$ which is contained in $\overline{B\left(x_{0}, 2 a\right)}$ and is of class $H^{1}$ (i.e. $t \mapsto \frac{d}{d t}(\alpha(t, x))$ is $\left.L^{2}\right)$, and provided the following conditions are satisfied:

(i) $u \in L^{2}\left(J_{b} ; \mathbb{U}\right)$,

(ii) there exists a linear map $M \in \mathcal{L}\left(\ell^{2}(\mathbb{N}) ; E\right)$ such that for every $x$ and $y$ belonging to $V$ we have

$$
\left|X_{i}^{\lambda}(x)-X_{i}^{\lambda}(y)\right| \leq M_{i}^{\lambda} d_{E}(x, y)
$$

where

$$
x=\sum_{\lambda} x^{\lambda} e_{\lambda}, \quad y=\sum_{\lambda} y^{\lambda} e_{\lambda} \quad \text { and } \quad d_{E}(x, y)=\sum_{\lambda}\left(x^{\lambda}-y^{\lambda}\right)^{2},
$$

(iii) the map $M \circ v: J_{b} \rightarrow E$ belongs to $L^{2}\left(J_{b}, E\right)$, where we use the notation $\left|u^{i}(t)\right|=v^{i}(t)$.

Remark 2.1. The set $\left\{X_{i}: i \in \mathbb{N}\right\}$ generates in $E$ a distribution $\mathcal{F}$ with typical fiber $F$. Conversely if $\mathcal{F}$ is a trivial fiber on $E$ with typical fiber $F$, there exists a family $\left\{X_{i}(x): i \in \mathbb{N}\right\}$ which is an orthonormal basis in each fiber. If we decompose $X_{i}=$ $\sum_{\lambda} X_{i}^{\lambda} e_{\lambda}$, for each $H^{1}$ curve $\gamma$ which is tangent to $\mathcal{F}$, there exists a family $\left(u_{i}\right)_{i \in \mathbb{N}} \in \mathbb{U}$ such that

$$
\dot{x}=\sum_{i} u_{i} X_{i}
$$


without assuming any additional conditions on the set $\left\{X_{i}: i \in \mathbb{N}\right\}$. Note that for a general sequence $\left(u_{i}\right)_{i \in \mathbb{N}} \in \mathbb{U}$ a curve which is a solution of $(D)$ does not always exist.

2.1.1. Bilinear Hilbert-Schmidt Distributions. Denote by $\mathcal{L}(E ; F)$ (resp. $\mathcal{L}_{H . S}(E ; F)$ ) the vector space of linear bounded operators (resp. Hilbert-Schmidt operators) from $E$ to $F$ and $\mathcal{L}(F ; \mathcal{L}(E ; F))$ (resp. $\left.\mathcal{L}_{H . S}\left(F ; \mathcal{L}_{H . S}(E ; F)\right)\right)$ the space of bounded operators (resp. Hilbert-Schmidt operators) from $F$ to $\mathcal{L}(E ; F)$ (resp. $\mathcal{L}_{H . S}(E ; F)$ ). Consider $A \in \mathcal{L}(F ; F)\left(u \mapsto A_{u}\right), B \in \mathcal{L}(F ; \mathcal{L}(E ; F))\left(u \mapsto B_{u}\right)$ and the operator $\widetilde{B} \in \mathcal{L}(F \times E ; F)$ associated to $B$ defined by

$$
\widetilde{B}(u, x)=B_{u} x \quad \text { for every } u \in F \text { and every } x \in E .
$$

Denote by $\left\{f_{i}: i \in \mathbb{N}\right\}$ a Hilbert basis for $F$ and set

$$
X_{i}(x)=A_{f_{i}}+B_{f_{i}} x .
$$

Consider the associated system $(\Sigma)$ defined by

$$
\dot{x}=A x+\widetilde{B}(u, x) .
$$

We call $(\Sigma)$ a bilinear system on $E$. If in addition $B \in \mathcal{L}_{H . S}\left(F ; \mathcal{L}_{H . S}(E ; F)\right)$ we say that $(\Sigma)$ is a bilinear Hilbert-Schmidt system.

Next, we denote by $\mathcal{F}$ the distribution generated by $\left\{X_{i}: i \in \mathbb{N}\right\}$ and call it a bilinear distribution. In case $B \in \mathcal{L}_{H . S}\left(F ; \mathcal{L}_{H . S}(E ; F)\right.$ we say that $\mathcal{F}$ is a bilinear Hilbert-Schmidt distribution.

Now, by application of Theorem 2.1 we have:

Lemma 2.1. If $\mathcal{F}$ is a bilinear Hilbert-Schmidt distribution then to each horizontal curve we can associate a control $u(t)$ and conversely.

Remark 2.2. For the previous family $\left\{X_{i}: i \in \mathbb{N}\right\}$ each Lie bracket $\left[X_{i}, X_{j}\right]$ is the constant vector field $\sum_{\lambda}\left\{A B_{f_{i}}\left(e_{\lambda}\right)-A B_{f_{j}}\left(e_{\lambda}\right)\right\}$. So, the Lie algebra generated by this family of vector fields is nilpotent of step 2 .

2.2. Bilinear distribution and Hilbert 2-step nilpotent Lie groups. Let $G$ be a Hilbertian Lie group and $\mathcal{G}$ its Lie algebra. If $[\cdot, \cdot]$ denotes the Lie bracket, the center of $\mathcal{G}$ is the largest subspace $\mathcal{Z}$ such that $[\mathcal{Z}, \mathcal{G}]=0$. Let $\mathcal{G}^{0}=\mathcal{G}, \mathcal{G}^{k}=\left[\mathcal{G}, \mathcal{G}^{k-1}\right]$ for $k \geq 1$ integer. Recall that a Lie algebra $\mathcal{G}$ is nilpotent if there exists $k \geq 1$ such that $\mathcal{G}^{k}=\{0\}$, and $\mathcal{G}$ is nilpotent of step $r$ if $\mathcal{G}^{r}=\{0\}$ with $\mathcal{G}^{r-1} \neq\{0\}$. A Hilbert Lie group is $r$-step nilpotent if and only if its Lie algebra is also $r$-step nilpotent.

Consider now a connected Hilbert 2-step nilpotent Lie group $G$ and let $\mathcal{Z}$ be the center of its Lie algebra $\mathcal{G}$, for which we have of course $\mathcal{Z} \supset \mathcal{G}^{1}$.

As $\mathcal{Z}$ is a closed Lie sub-algebra of $\mathcal{G}$ we have

$$
\mathcal{G}=F \oplus \mathcal{Z}
$$

where $F=\mathcal{Z}^{\perp}$ is the orthogonal complement of $\mathcal{Z}$ with respect to the Hilbert inner product defined on $\mathcal{G}$. The Lie bracket gives rise to a bilinear skew-symmetric map 
$\Lambda: \mathcal{G} \times \mathcal{G} \rightarrow \mathcal{G}$ which satisfies the Jacobi identity, that is

$$
\Lambda(\Lambda(u, v), w)+\Lambda(\Lambda(v, w), u)+\Lambda(\Lambda(w, u), v)=0 \quad \text { for all } u, v, w \in \mathcal{G}
$$

and we have $\operatorname{Im} \Lambda \subset \mathcal{Z}$. If $\mathcal{G}$ is 2 -step nilpotent then the Jacobi identity is trivially satisfied. On the other hand if $\Lambda$ is such a skew-symmetric map on a Hilbert space $\mathcal{G}$ we have a unique 2-step nilpotent Lie algebra associated structure on $\mathcal{G}$.

The exponential map exp : $\mathcal{G} \rightarrow G$ is $\mathcal{C}^{\infty}$-surjective and is a diffeomorphism from a neighborhood of 0 onto a neighborhood of the identity. This map allows us to identify a neighborhood of the identity $G$ with an open set $U$ in $\mathcal{G}$ which contains 0 . In this chart we have $d_{x} \exp (u)=u+\frac{1}{2}[x, u]$.

For each $u \in F$ and $x \in \mathcal{G}$ there exists a unique operator $B \in \mathcal{L}(F \times \mathcal{G} ; F)$ defined by

$$
\langle v, B(u, x)\rangle=\langle[u, v], x\rangle=\langle\Lambda(u, v), x\rangle \quad \forall v \in F .
$$

The left-invariant distribution $\mathcal{F}$ on $U$ induced by $F$ is then equal to $d \exp (F)$. It is the bilinear distribution associated to $A=\mathrm{Id}_{F}$ and $B$ as defined in Subsection 2.1.1 for system $(\Sigma)$. Moreover, if $\Lambda$ is a Hilbert-Schmidt operator then $B$ is also a Hilbert-Schmidt operator and the associated distribution is a bilinear Hilbert-Schmidt distribution.

Definition 2.1. We say that $G$ is a 2-step nilpotent Hilbert-Schmidt Lie group if the operator $\Lambda$ of its Lie algebra is a Hilbert-Schmidt operator.

Remark 2.3. Denote by $\left\{f_{i}: i \in \mathbb{N}\right\}$ a Hilbert basis for a subspace $F$ of $E$. If we set $X_{i}(x)=f_{i}+B_{f_{i}} x$ where $B \in \mathcal{L}(F ; \mathcal{L}(E ; F))$, and denote by $\mathcal{F}$ the bilinear distribution on $E$ generated by the family $X_{i}$, we can define a Lie group structure on $E$ given by the following product:

$$
\begin{gathered}
\mathcal{G} \times \mathcal{G} \rightarrow \mathcal{G}, \\
(u, v) \mapsto u \bullet v=u+v+\frac{1}{2}[u, v] .
\end{gathered}
$$

The Lie algebra of this structure is of course $E$ with the Lie bracket structure defined by $\left[f_{i}, f_{j}\right]=\left[X_{i}, X_{j}\right]$ which is isomorphic to the Lie algebra associated to $\mathcal{F}$ (see Remark 2.2). It is easy to see that for this structure of Lie group on $E$, the left-invariant distribution defined by $F$ is precisely $\mathcal{F}$.

So the study of bilinear systems for which the linear operator $A$ is Hilbert-Schmidt and invertible is equivalent to the study of left-invariant distributions on 2-step nilpotent Hilbert Lie groups.

\section{Accessibility problems on 2-step nilpotent Hilbert Lie groups}

3.1. Introduction. The purpose of this section is to study accessibility problems on a connected 2-step nilpotent Hilbert Lie group.

Let $G$ be such a group and $\mathcal{G}$ its Lie algebra. Recall that we have the decomposition

$$
\mathcal{G}=F \oplus \mathcal{Z}
$$

where $\mathcal{Z}$ is the center of $\mathcal{G}$ and $F$ is orthogonal to $\mathcal{Z}$.

Further, denote by $\Lambda: F \times F \rightarrow \mathcal{Z}$ the continuous skew-symmetric bilinear operator which induces the Lie bracket and by $\operatorname{Im} \Lambda$ the image of $\Lambda$. 
Let $\mathcal{H}$ be a closed Lie algebra generated by $F$ in $\mathcal{G}$. Obviously we have

$$
\mathcal{H}=F \oplus \overline{\operatorname{Im} \Lambda} \text {. }
$$

One will denote by $H$ the connected Lie subgroup $G$ whose Lie algebra is $\mathcal{H}$. One then has:

THEOREM 3.1. There is a neighborhood $U$ of the identity e and a subset $U_{0}$ dense in $H \cap U$ such that for every $g \in G$ and for every $h \in g U_{0}$ there is a horizontal path of class $H^{1}$ (i.e. with derivative of class $L^{2}$ ) which joins $h$ to $g$. This path is in fact contained in $\mathrm{gH}$.

Theorem 3.2. Assume $\Lambda$ is a Hilbert-Schmidt operator and $\operatorname{Im} \Lambda$ is of finite dimension. Then for every $g \in G$ and for every $h \in g H$ there is a horizontal absolutely continuous path (i.e. with derivative of class $L^{1}$ ) which joins $g$ and $h$ (this path is contained in $g H)$. Conversely, if two points $g$ and $h$ are joined by a horizontal path then $h \in g H$.

COROLlary 3.1 (Chow's theorem).

1) If $\overline{\operatorname{Im} \Lambda}=\mathcal{Z}$ then there is a neighborhood $U$ of the identity and a subset $U_{0}$ dense in $U$ such that for every $h \in g U_{0}$ there is a horizontal path of class $H^{1}$ joining $h$ and $g$.

2) Moreover, if $\mathcal{Z}$ is of finite dimension and $\Lambda$ is Hilbert-Schmidt then for every $g$ and $h$ belonging to $G$ there is a horizontal absolutely continuous path joining $g$ and $h$.

First we are going to establish a local version of these theorems in the following subsection.

3.2. Preliminary results. Let $\left\{f_{i}: i \in \mathbb{N}\right\}$ (resp. $\left\{v_{\beta}: \beta \in \mathbb{N}\right\}$ ) be a Hilbert basis for $F$ (resp. for $\mathcal{Z}$ ). There is a non-unique family of pairs of indices $\left\{\left(i_{\alpha}, j_{\alpha}\right): \alpha \in \mathbb{N}\right\}$ such that if we put

$$
z_{i_{\alpha} j_{\alpha}}=\left[f_{i_{\alpha}}, f_{j_{\alpha}}\right]
$$

then $\left\{z_{i_{\alpha} j_{\alpha}}: \alpha \in \mathbb{N}\right\}$ is a topological basis of $\mathcal{Z}$. More precisely, for every $\alpha$ of $\mathbb{N}$ there is $\left(C_{i_{\alpha} j_{\alpha}}^{\mu}\right) \in \ell^{2}(\mathbb{N})$ such that

$$
z_{i_{\alpha} j_{\alpha}}=\sum_{\mu} C_{i_{\alpha} j_{\alpha}}^{\mu} v_{\mu}
$$

with $C_{i_{\alpha} j_{\alpha}}^{\mu}=-C_{j_{\alpha} i_{\alpha}}^{\mu}$ for all $\mu \in \mathbb{N}$.

As in Remark 2.3, we can define a Lie structure on $\mathcal{G}$. Let us denote by $\widetilde{G}$ the corresponding Lie group. It is well known that there exists a diffeomorphism from a neighborhood of the identity of $G$ onto a neighborhood of the identity of $\widetilde{G}$. So without loss of generality we will suppose that $G=\widetilde{G}$.

We prove the following result:

THeOREM 3.3. There exists a subset $\widetilde{D}$ dense in $\widetilde{G}$ such that for every $y$ belonging to $\widetilde{D}$ there is a horizontal path of class $H^{1}$ joining y to the identity element of $\widetilde{G}$. More if $\Lambda$ is Hilbert-Schmidt and the center of $\mathcal{G}$ is of finite dimension, then all points of $\widetilde{G}$ can be joined to the identity of $\widetilde{G}$ by a horizontal absolutely continuous path. 
We denote by $\prod$ the finite or infinite product with respect to the operation $\bullet$

LEMMA 3.1. Let $u=\sum_{i} x_{i} f_{i}+\sum_{\alpha} \lambda_{\alpha} z_{i_{\alpha} j_{\alpha}}$ be an element of $\mathcal{G}$ such that

$$
\sum_{\mu}\left(\sum_{i<j} C_{i j}^{\mu} x_{i} x_{j}\right)^{2}<\infty
$$

then

$$
\Psi(u)=\prod_{\alpha} \phi_{z_{i_{\alpha} j_{\alpha}}}\left(\lambda_{\alpha}\right) \bullet \prod_{i} \phi_{f_{i}}\left(x_{i}\right)
$$

is well defined. Moreover, if $\Lambda$ is a Hilbert-Schmidt operator then $\Psi$ is a local diffeomorphism from $\mathcal{G}$ to $\widetilde{G}$ which is surjective.

Proof. It is easy to see that $\Psi$ writes in the basis $\left(f_{i}, v_{\mu}\right)$ as follows:

$$
\begin{aligned}
& \Psi(u)=\sum_{i} x_{i} f_{i}+\sum_{\mu} \sum_{\alpha} C_{i_{\alpha} j_{\alpha}}^{\mu} \lambda_{\alpha} v_{\mu}+\frac{1}{2} \sum_{\mu} \sum_{i<j} C_{i j}^{\mu} x_{i} x_{j} v_{\mu} \\
&=u+\frac{1}{2} \sum_{\mu} \sum_{i<j} C_{i j}^{\mu} x_{i} x_{j} v_{\mu} .
\end{aligned}
$$

The first assertion is thus obvious. Suppose that $\Lambda$ is a Hilbert-Schmidt operator. Let us denote by $A: F \times F \rightarrow \mathcal{Z}$ the operator defined by

$$
A(x, y)=\frac{1}{2} \sum_{\mu} \sum_{i<j} C_{i j}^{\mu} x_{i} y_{j} v_{\mu}
$$

with $x=\sum_{i} x_{i} f_{i}$ and $y=\sum_{j} y_{j} f_{j}$. Then $A$ is a well defined bilinear Hilbert-Schmidt operator. When identifying $\mathcal{G}$ with $F \times \mathcal{Z}$ one has for $(x, z) \in F \times \mathcal{Z}$

$$
\Psi((x, z))=x+z+A(x, x) .
$$

Therefore, $\Psi$ is $\mathcal{C}^{\infty}$. In addition, the differential of $\Psi$ at $(0,0)$ is

$$
D_{(0,0)} \Psi=\left(\begin{array}{cc}
\text { Id } & 0 \\
0 & \text { Id }
\end{array}\right) \text {. }
$$

Thus $\Psi$ is a local diffeomorphism. However if $y \in \widetilde{G}$ with $y=\sum_{i} y_{i} f_{i}+\sum_{\alpha} b_{\alpha} v_{\alpha}$ then

$$
u=\sum_{i} y_{i} f_{i}+\sum_{\alpha}\left(b_{\alpha}-\frac{1}{2} \sum_{i<j} C_{i j}^{\alpha} y_{i} y_{j}\right) v_{\alpha}
$$

is well defined and satisfies $\Psi(u)=y$.

3.3. Construction of paths. Let $u=\sum_{i} x_{i} f_{i}+\sum_{\alpha} \lambda_{\alpha} z_{i_{\alpha} j_{\alpha}}$ be an element of $\mathcal{G}$ such that $\sum_{\mu}\left(\sum_{i<j} C_{i j}^{\mu} x_{i} x_{j}\right)^{2}<\infty$ and $\sum_{\alpha}\left|\lambda_{\alpha}\right|^{2}<\infty$ (this last condition holds for example if $\left\{z_{i_{\alpha} j_{\alpha}}: \alpha \in \mathbb{N}\right\}$ is a Hilbert basis). We shall build an absolutely continuous path $\gamma_{u}:[0,1] \rightarrow \widetilde{G}$ joining the identity of $\widetilde{G}$ to $\Psi(u)$. 
Step 1. Let $\|u\|_{F}^{2}=\sum_{i}\left|x_{i}\right|^{2}$. If $\|u\|_{F}=0$, just consider the constant path $\gamma_{u}(t)=0$ (identity of $\widetilde{G}$ ) for $0 \leq t \leq \frac{1}{2}$. Suppose that $\|u\|_{F} \neq 0$.

Define the sequence $\left(r_{i}\right)_{i \in \mathbb{N}}$ in $\left[0, \frac{1}{2}\right]$ by

$$
r_{0}=0, \quad r_{i}-r_{i-1}=\frac{\left|x_{i}\right|^{2}}{2\|u\|_{F}^{2}} \quad \text { if } i>0,
$$

then $\sum_{i}\left(r_{i}-r_{i-1}\right)=\frac{1}{2}=\lim _{i \rightarrow \infty} r_{i}$.

If $x_{i} \neq 0$, then for all $t \in\left[r_{i-1}, r_{i}\right]$ we define the path

$$
\gamma_{u}(t)=\phi_{f_{i}}\left(2\left(t-r_{i-1}\right) \frac{\|u\|_{F}^{2}}{\left|x_{i}\right|^{2}} x_{i}\right) \bullet \phi_{f_{i-1}}\left(x_{i-1}\right) \bullet \ldots \bullet \phi_{f_{1}}\left(x_{1}\right)
$$

for which it is clear that

$$
\gamma_{u}(0)=0
$$

Let

which is well defined because

$$
\gamma_{u}\left(\frac{1}{2}\right)=\lim _{i \rightarrow \infty} \gamma_{u}\left(r_{i}\right)
$$

$$
\lim _{i \rightarrow \infty} \gamma_{u}\left(r_{i}\right)=\prod_{i \in \mathbb{N}} \phi_{f_{i}}\left(x_{i}\right)=\sum_{i} x_{i} f_{i}+\frac{1}{2} \sum_{\mu} \sum_{i<j} C_{i j}^{\mu} x_{i} x_{j} v_{\mu} .
$$

Let us show that

$$
\lim _{t \rightarrow \frac{1}{2}} \gamma_{u}(t)=\gamma_{u}\left(\frac{1}{2}\right)
$$

Taking $i$ such that $t \in\left[r_{i-1}, r_{i}\right]$ we have

$$
\left\|\gamma_{u}(t)-\gamma_{u}\left(\frac{1}{2}\right)\right\| \leq\left\|\gamma_{u}(t)-\gamma_{u}\left(r_{i-1}\right)\right\|+\left\|\gamma_{u}\left(r_{i-1}\right)-\gamma_{u}\left(\frac{1}{2}\right)\right\| .
$$

However

$$
\begin{aligned}
\gamma_{u}\left(r_{i-1}\right)=\phi_{f_{1}}\left(x_{1}\right) \bullet \ldots \bullet \phi_{f_{i-1}} & \left(x_{i-1}\right) \\
& =x_{1} f_{1}+\ldots+x_{i-1} f_{i-1}+\frac{1}{2} \sum_{1 \leq j<k<i} \sum_{\mu} C_{j k}^{\mu} x_{j} x_{k} v_{\mu}
\end{aligned}
$$

and

$$
\begin{aligned}
\gamma_{u}(t)=x_{1} f_{1}+\ldots & +x_{i-1} f_{i-1}+2\left(t-r_{i-1}\right) \frac{\|u\|_{F}^{2}}{\left|x_{i}\right|^{2}} x_{i} f_{i} \\
& +\frac{1}{2} \sum_{1 \leq j<k<i} \sum_{\mu} C_{j k}^{\mu} x_{j} x_{k} v_{\mu}+\sum_{h=1}^{i-1} \sum_{\mu} C_{i h}^{\mu}\left(t-r_{i-1}\right) \frac{\|u\|_{F}^{2}}{\left|x_{i}\right|^{2}} x_{i} x_{h} v_{\mu} .
\end{aligned}
$$

Thus

$$
\gamma_{u}(t)-\gamma_{u}\left(r_{i-1}\right)=2\left(t-r_{i-1}\right) \frac{\|u\|_{F}^{2}}{\left|x_{i}\right|^{2}} x_{i} f_{i}+\sum_{h=1}^{i-1} \sum_{\mu} C_{i h}^{\mu}\left(t-r_{i-1}\right) \frac{\|u\|_{F}^{2}}{\left|x_{i}\right|^{2}} x_{i} x_{h} v_{\mu} .
$$


If we denote by $\|\Lambda\|$ the norm of the continuous bilinear map $\Lambda$, we get

$$
\begin{aligned}
\left\|\gamma_{u}(t)-\gamma_{u}\left(r_{i-1}\right)\right\| & \leq 2\left(t-r_{i-1}\right) \frac{\|u\|_{F}^{2}}{\left|x_{i}\right|}+\left\|\sum_{h=1}^{i-1} \sum_{\mu} C_{i h}^{\mu}\left(t-r_{i-1}\right) \frac{\|u\|_{F}^{2}}{\left|x_{i}\right|^{2}} x_{i} x_{h} v_{\mu}\right\| \\
& \leq 2\left(t-r_{i-1}\right) \frac{\|u\|_{F}^{2}}{\left|x_{i}\right|}+\left\|\Lambda\left(\left(t-r_{i-1}\right) \frac{\|u\|_{F}^{2}}{\left|x_{i}\right|^{2}} x_{i} f_{i}, \sum_{h=1}^{i-1} x_{h} f_{h}\right)\right\| \\
& \leq 2\left(t-r_{i-1}\right) \frac{\|u\|_{F}^{2}}{\left|x_{i}\right|}+\|\Lambda\|\left(t-r_{i-1}\right) \frac{\|u\|_{F}^{2}}{\left|x_{i}\right|}\left\|\sum_{h=1}^{i-1} x_{h} f_{h}\right\|_{L^{2}} \\
& \leq 2\left(t-r_{i-1}\right) \frac{\|u\|_{F}^{2}}{\left|x_{i}\right|}+\left(t-r_{i-1}\right) \frac{\|u\|_{F}^{2}}{\left|x_{i}\right|}\|\Lambda\|\left(\sum_{h}\left|x_{h}\right|^{2}\right)^{\frac{1}{2}} .
\end{aligned}
$$

Finally one has the estimate

$$
\left\|\gamma_{u}(t)-\gamma_{u}\left(\frac{1}{2}\right)\right\| \leq\left|x_{i}\right|\left(1+\frac{1}{2}\|\Lambda\|\|u\|_{F}\right)+\left\|\gamma_{u}\left(r_{i-1}\right)-\gamma_{u}\left(\frac{1}{2}\right)\right\| .
$$

Since $\lim _{i \rightarrow \infty}\left|x_{i}\right|=0$ (because $\left.\sum_{i}\left|x_{i}\right|^{2}<\infty\right)$ and $\gamma_{u}\left(\frac{1}{2}\right)=\lim _{i \rightarrow \infty} \gamma_{u}\left(r_{i}\right)$, the result is proved.

Step 2. Let us define $\|\lambda\|_{\mathcal{Z}}^{2}=\sum_{\alpha}\left|\lambda_{\alpha}\right|^{2}$. If $\|\lambda\|_{\mathcal{Z}}=0$, we consider $\gamma_{u}(t)=\gamma_{u}\left(\frac{1}{2}\right)$ for $\frac{1}{2} \leq t \leq 1$. Suppose that $\|\lambda\|_{\mathcal{Z}} \neq 0$.

Define in $\left[\frac{1}{2}, 1\right]$ the sequence $\left(s_{\alpha}\right)_{\alpha \in \mathbb{N}}$ by

$$
s_{0}=\frac{1}{2}, \quad s_{\alpha}= \begin{cases}s_{\alpha-1}+\frac{\left|\lambda_{\alpha}\right|^{2}}{2\|\lambda\|_{\mathcal{Z}}^{2}} & \text { if } \alpha>0, \lambda_{\alpha} \neq 0, \\ s_{\alpha-1} & \text { if } \alpha>0, \lambda_{\alpha}=0,\end{cases}
$$

and consider the subdivision of the interval $\left[s_{\alpha-1}, s_{\alpha}\right]$ given by

$$
\begin{aligned}
I_{\alpha}^{1} & =\left[s_{\alpha-1}, s_{\alpha-1}+\frac{1}{4}\left(s_{\alpha}-s_{\alpha-1}\right)\right], \\
I_{\alpha}^{2} & =\left[s_{\alpha-1}+\frac{1}{4}\left(s_{\alpha}-s_{\alpha-1}\right), s_{\alpha-1}+\frac{1}{2}\left(s_{\alpha}-s_{\alpha-1}\right)\right], \\
I_{\alpha}^{3} & =\left[s_{\alpha-1}+\frac{1}{2}\left(s_{\alpha}-s_{\alpha-1}\right), s_{\alpha-1}+\frac{3}{4}\left(s_{\alpha}-s_{\alpha-1}\right)\right], \\
I_{\alpha}^{4} & =\left[s_{\alpha-1}+\frac{3}{4}\left(s_{\alpha}-s_{\alpha-1}\right), s_{\alpha}\right] .
\end{aligned}
$$

For $t \in\left[s_{\alpha-1}, s_{\alpha}\right]$ we define the path

$$
\gamma_{u}(t)=\Gamma_{i_{\alpha} j_{\alpha}}^{s_{\alpha-1} s_{\alpha}}\left(\lambda_{\alpha}\right)(t) \bullet \phi_{z_{i_{\alpha}-1} j_{\alpha-1}}\left(\lambda_{\alpha-1}\right) \bullet \ldots \bullet \phi_{z_{i_{1} j_{1}}}\left(\lambda_{1}\right) \bullet \prod_{i \in \mathbb{N}} \phi_{f_{i}}\left(x_{i}\right)
$$

where $\Gamma$ is the path defined for every $\lambda_{\alpha} \geq 0$ as follows:

$$
\begin{array}{ll}
\Gamma_{i_{\alpha} j_{\alpha}}^{s_{\alpha-1} s_{\alpha}}\left(\lambda_{\alpha}\right)(t)=\phi_{f_{j_{\alpha}}}\left(\left(t-s_{\alpha-1}\right) \frac{4 \lambda_{\alpha}^{\frac{1}{2}}}{s_{\alpha}-s_{\alpha-1}}\right) & \text { if } t \in I_{\alpha}^{1}, \\
\Gamma_{i_{\alpha} j_{\alpha}}^{s_{\alpha-1} s_{\alpha}}\left(\lambda_{\alpha}\right)(t)=\phi_{f_{i_{\alpha}}}\left(\left(t-s_{\alpha-1}-\frac{1}{4}\left(s_{\alpha}-s_{\alpha-1}\right)\right) \frac{4 \lambda_{\alpha}^{\frac{1}{2}}}{s_{\alpha}-s_{\alpha-1}}\right) \bullet \phi_{f_{j_{\alpha}}}\left(\lambda_{\alpha}^{\frac{1}{2}}\right) & \text { if } t \in I_{\alpha}^{2},
\end{array}
$$




$$
\begin{aligned}
\Gamma_{i_{\alpha} j_{\alpha}}^{s_{\alpha-1} s_{\alpha}}\left(\lambda_{\alpha}\right)(t)=\phi_{f_{j_{\alpha}}} & \left(-\left(t-s_{\alpha-1}-\frac{3}{4}\left(s_{\alpha}-s_{\alpha-1}\right)\right) \frac{4 \lambda_{\alpha}^{\frac{1}{2}}}{s_{\alpha}-s_{\alpha-1}}\right) & & \\
& \bullet \phi_{f_{i_{\alpha}}}\left(\lambda_{\alpha}^{\frac{1}{2}}\right) \bullet \phi_{f_{j_{\alpha}}}\left(\lambda_{\alpha}^{\frac{1}{2}}\right) & & \text { if } t \in I_{\alpha}^{3}, \\
\Gamma_{i_{\alpha} j_{\alpha}}^{s_{\alpha-1} s_{\alpha}}\left(\lambda_{\alpha}\right)(t)=\phi_{f_{i_{\alpha}}} & \left(-\left(t-s_{\alpha-1}-\frac{3}{4}\left(s_{\alpha}-s_{\alpha-1}\right)\right) \frac{4 \lambda_{\alpha}^{\frac{1}{2}}}{s_{\alpha}-s_{\alpha-1}}\right) & & \\
& \bullet \phi_{f_{j_{\alpha}}}\left(-\lambda_{\alpha}^{\frac{1}{2}}\right) \bullet \phi_{f_{i_{\alpha}}}\left(\lambda_{\alpha}^{\frac{1}{2}}\right) \bullet \phi_{f_{j_{\alpha}}}\left(\lambda_{\alpha}^{\frac{1}{2}}\right) & & \text { if } t \in I_{\alpha}^{4},
\end{aligned}
$$

and for $\lambda_{\alpha}<0$ we set

$$
\Gamma_{i_{\alpha} j_{\alpha}}^{s_{\alpha-1} s_{\alpha}}\left(\lambda_{\alpha}\right)(t)=\Gamma_{j_{\alpha} i_{\alpha}}^{s_{\alpha-1} s_{\alpha}}\left(\left|\lambda_{\alpha}\right|\right)(t)
$$

One can easily check that for every $\lambda_{\alpha}$ positive or negative we have

$$
\Gamma_{i_{\alpha} j_{\alpha}}^{s_{\alpha-1} s_{\alpha}}\left(\lambda_{\alpha}\right)\left(s_{\alpha}\right)=\phi_{z_{i_{\alpha} j_{\alpha}}}\left(\lambda_{\alpha}\right) .
$$

Let

This is well defined because

$$
\gamma_{u}(1)=\lim _{\alpha \rightarrow \infty} \gamma_{u}\left(s_{\alpha}\right)
$$

$$
\begin{aligned}
\lim _{\alpha \rightarrow \infty} \gamma_{u}\left(s_{\alpha}\right)=\prod_{\alpha \in \mathbb{N}} \phi_{z_{i_{\alpha} j_{\alpha}}}\left(\lambda_{\alpha}\right) \bullet \prod_{i \in \mathbb{N}} \phi_{f_{i}}\left(x_{i}\right) \\
\quad=\sum_{i} x_{i} f_{i}+\sum_{\alpha} \lambda_{\alpha} z_{i_{\alpha} j_{\alpha}}+\frac{1}{2} \sum_{\mu} \sum_{i<j} C_{i j}^{\mu} x_{i} x_{j} v_{\mu}=\Psi(u) .
\end{aligned}
$$

Let us show that

$$
\lim _{t \rightarrow 1} \gamma_{u}(t)=\gamma_{u}(1)
$$

Fix $t \in\left[\frac{1}{2}, 1\right]$, then there is $t \in\left[s_{\alpha-1}, s_{\alpha}\right]$ such that

$$
\left\|\gamma_{u}(t)-\gamma_{u}(1)\right\| \leq\left\|\gamma_{u}(t)-\gamma_{u}\left(s_{\alpha-1}\right)\right\|+\left\|\gamma_{u}\left(s_{\alpha-1}\right)-\gamma_{u}(1)\right\| .
$$

with

$$
\begin{aligned}
\gamma_{u}\left(s_{\alpha-1}\right)=\phi_{z_{i_{\alpha-1} j_{\alpha-1}}}\left(\lambda_{\alpha-1}\right) & \bullet \ldots \bullet \phi_{z_{i_{1} j_{1}}}\left(\lambda_{1}\right) \bullet \prod_{i \in \mathbb{N}} \phi_{f_{i}}\left(x_{i}\right) \\
& =\sum_{i} x_{i} f_{i}+\sum_{\mu} \sum_{l=1}^{\alpha-1} C_{i_{l} j_{l}}^{\mu} \lambda_{l} v_{\mu}+\frac{1}{2} \sum_{\mu} \sum_{i<j} C_{i j}^{\mu} x_{i} x_{j} v_{\mu}
\end{aligned}
$$

and

$$
\gamma_{u}(t)=\Gamma_{i_{\alpha} j_{\alpha}}^{s_{\alpha-1} s_{\alpha}}\left(\lambda_{\alpha}\right)(t) \bullet\left(\sum_{i} x_{i} f_{i}+\sum_{\mu} \sum_{l=1}^{\alpha-1} C_{i_{l} j_{l}}^{\mu} \lambda_{l} v_{\mu}+\frac{1}{2} \sum_{\mu} \sum_{i<j} C_{i j}^{\mu} x_{i} x_{j} v_{\mu}\right) .
$$

We then have four cases:
1) $t \in I_{\alpha}^{1}=\left[s_{\alpha-1}, s_{\alpha-1}+\frac{1}{4}\left(s_{\alpha}-s_{\alpha-1}\right)\right]$,
2) $t \in I_{\alpha}^{2}=\left[s_{\alpha-1}+\frac{1}{4}\left(s_{\alpha}-s_{\alpha-1}\right), s_{\alpha-1}+\frac{1}{2}\left(s_{\alpha}-s_{\alpha-1}\right)\right]$,
3) $t \in I_{\alpha}^{3}=\left[s_{\alpha-1}+\frac{1}{2}\left(s_{\alpha}-s_{\alpha-1}\right), s_{\alpha-1}+\frac{3}{4}\left(s_{\alpha}-s_{\alpha-1}\right)\right]$,
4) $t \in I_{\alpha}^{4}=\left[s_{\alpha-1}+\frac{3}{4}\left(s_{\alpha}-s_{\alpha-1}\right), s_{\alpha}\right]$. 
By arguments analogous with the ones of Step 1 we show that there is a constant $M$ independent of $t \in\left[s_{\alpha-1}, s_{\alpha}\right]$ such that

$$
\left\|\gamma_{u}(t)-\gamma_{u}(1)\right\| \leq \lambda_{\alpha}^{\frac{1}{2}} M+\left\|\gamma_{u}\left(s_{\alpha-1}\right)-\gamma_{u}(1)\right\|
$$

Since $\lim _{\alpha \rightarrow \infty} \lambda_{\alpha}^{\frac{1}{2}}=0$ (because $\left.\sum_{\alpha} \lambda_{\alpha}^{2}<\infty\right)$ and we know that $\gamma_{u}(1)=\lim _{\alpha \rightarrow \infty} \gamma_{u}\left(s_{\alpha}\right)$ the result is proved.

Thus, the path has been built and by construction, it is absolutely continuous.

\subsection{Proof of the results}

3.4.1. Proof of TheOrem 3.3. For each $u \in \mathcal{G}$ consider the decomposition $u=$ $\sum_{i} x_{i} f_{i}+\sum_{\alpha} \lambda_{\alpha} v_{\alpha}$. Let $D$ be the set of points $u$ of $\mathcal{G}$ such that there is a finite number of indices $i$ with $x_{i} \neq 0$ and a finite number of indices $\alpha$ with $\lambda_{i_{\alpha} j_{\alpha}} \neq 0$. Then it is clear that $\Psi(D)$ is a dense subset of $\widetilde{G}$. Moreover, for any $u \in D$ we have $\sum_{\mu}\left(\sum_{i<j} C_{i j}^{\mu} x_{i} x_{j}\right)^{2}<\infty$ because $\left|C_{i j}^{\mu}\right|<\|\Lambda\|$ and there is a finite number of $x_{i} \neq 0$. We also have $\sum_{\alpha} \lambda_{\alpha}^{2}<\infty$.

The assumptions of Subsection 3.3 are then satisfied. We can also build a path joining any element $y \in \Psi(D)$ to the identity in $\widetilde{G}$. It remains to be shown that this path is of class $H^{1}$.

On one hand for every $t \in\left[0, \frac{1}{2}\right]$, there is an index $j$ such that $t$ belongs to $\left[r_{j-1}, r_{j}\right]$ and we can define a sequence $w=\left(w_{i}\right)_{i \in \mathbb{N}}$ by

$$
w_{j}(t)= \begin{cases}2 \frac{\|u\|_{F}^{2}}{\left|x_{j}\right|^{2}} x_{j} & \text { if } x_{j} \neq 0 \text { and } t \in\left[r_{j-1}, r_{j}\right] \\ 0 & \text { otherwise. }\end{cases}
$$

Then

$$
\int_{0}^{\frac{1}{2}}\|w(t)\|^{2} d t=2 \sum_{\left\{j: j \geq 1, x_{j} \neq 0\right\}}\|u\|_{F}^{2} .
$$

As there is a finite number of indices $j$ such that $x_{j} \neq 0$, the last expression is finite.

On the other hand, for every $t \in\left[\frac{1}{2}, 1\right]$ there is an index $\beta$ such that $t$ belongs to $I(\beta)$ where $I(\beta)$ is one of the four intervals previously defined. We introduce a sequence $w=\left(w_{\alpha}\right)_{\alpha \in \mathbb{N}}$ by

$$
w_{\beta}(t)= \begin{cases}\frac{4 \lambda_{\beta}^{\frac{1}{2}}}{s_{\beta}-s_{\beta-1}} & \text { if } s_{\beta}-s_{\beta-1} \neq 0, \lambda_{\beta} \neq 0, t \in I(\beta) \\ 0 & \text { otherwise. }\end{cases}
$$

Then

$$
\int_{\frac{1}{2}}^{1}\|w(t)\|^{2} d t=32\|\lambda\|_{\mathcal{Z}}^{2} \sum_{\left\{\beta: \beta \geq 1, \lambda_{\beta} \neq 0\right\}} \frac{1}{\lambda_{\beta}}
$$

and since by assumption, there is a finite number of indices $\beta$ such that $\lambda_{\beta} \neq 0$, the last expression is finite, and then the proof of the general case is complete.

Suppose that $\Lambda$ is Hilbert-Schmidt and the center of $\mathcal{G}$ is a finite-dimensional space. Then the map $\Psi: \mathcal{G} \rightarrow \widetilde{G}$ is $\mathcal{C}^{\infty}$ surjective (Lemma 3.1). Take $y \in \widetilde{G}$ and $u \in \mathcal{G}$ such that 
$\Psi(u)=y$. We have $u=\sum_{i} x_{i} f_{i}+\sum_{\alpha} \lambda_{\alpha} v_{\alpha}$. Since $\Lambda$ is a Hilbert-Schmidt operator and $\operatorname{dim} \mathcal{Z}$ is finite, the assumptions of the last paragraph are satisfied: the horizontal path $\gamma_{u}$ associated to $u$ joins the identity to $\Psi(u)$. By construction, this path is absolutely continuous (but not $H^{1}$ in general).

3.4.2. Proof of Theorem 3.1 And the first part of Chow's theorem. Let $G$ be a Hilbertian 2-step nilpotent Lie group and $\mathcal{G}=F \oplus \mathcal{Z}$ its Lie algebra. Since for every $g$ the left translation $L_{g}$ takes the horizontal path, joining the identity $e$ to $h$, into a horizontal path joining $g$ at $L_{g}(h)$, it is enough to show the theorem with $g=e$.

Let $H$ be the connected Lie subgroup whose Lie algebra is $\mathcal{H}=F \oplus \overline{\operatorname{Im} \Lambda}$. As there exists a diffeomorphism $\theta$ from a neighborhood of the identity in $\widetilde{G}$ onto a neighborhood of the identity in $G$, by restriction, $\theta$ induces a diffeomorphism from a neighborhood of the identity in $\widetilde{H}$ onto a neighborhood of the identity in $H$, if $\widetilde{H}$ is the Lie group structure constructed on the Lie algebra $H$ as in Subsection 3.2. Theorem 3.1 and the first part of Chow's theorem are proved by application of Theorem 3.3 to $\mathcal{H}$ and $\widetilde{H}$ via $\theta$.

3.4.3. Proof of Theorem 3.2 And the SECond part of Chow's theorem. We have to prove our result for $g=e$.

Again we use the local diffeomorphism $\theta$ between $\widetilde{G}$ and $G$ mentioned in the previous subsection. As it induces a local diffeomorphism between $\widetilde{H}$ and $H$, we can apply Theorem 3.3 to $\widetilde{H}$ and then we get a neighborhood $U$ of $e$ in $H$ such that every point $h \in H$ can be joined to $e$ by a horizontal absolutely continuous path.

Now if $h$ is an arbitrary point of $H$, we consider a continuous path $c:[0,1] \rightarrow H$ joining $e$ to $h$ in $H$ ( $H$ is supposed to be connected). From the open covering $L_{c(t)}(U)$ of $c([0,1])$ we can extract a finite subcover

$$
V_{i}=L_{c\left(t_{i}\right)}(U), \quad 0 \leq t_{0} \leq t_{1} \leq \ldots \leq t_{N}=1
$$

Then take the points:

$$
\begin{gathered}
h_{0}=e, h_{1} \in V_{0} \cap V_{1}, h_{2}=c\left(t_{1}\right), h_{3} \in V_{1} \cap V_{2}, \ldots, \\
h_{2 i}=c\left(t_{i}\right), h_{2 i+1} \in V_{i} \cap V_{i+1}, \ldots, h_{2 N}=h .
\end{gathered}
$$

From Theorem 3.1 there is a horizontal absolutely continuous path $\gamma_{i}$ joining $h_{i}$ to $h_{i+1}, i=0, \ldots, 2 N$. By concatenation we obtain a horizontal absolutely continuous path $\gamma$ joining $e$ to $h$. Theorem 3.2 is then proved. For the second part of Chow's theorem we only have to take $\mathcal{H}=\mathcal{G}$ and $H=G$.

Conversely, if two points $g$ and $h$ are joined by a horizontal path $\gamma$ then $L_{g}^{-1}(\gamma)$ is a horizontal path joining $e$ to $h^{\prime}=L_{g}^{-1}(h)$.

Let $\Gamma$ be the characteristic subgroup of $\gamma$ (see next section). We then have $L_{g}^{-1}(\gamma) \subset$ $\Gamma \subset H$ and therefore $\gamma=L_{g} L_{g}^{-1}(\gamma)$ is contained in $L_{g}(H)$.

\section{Localization of abnormal curves}

4.1. Introduction. Consider a distribution $\mathcal{F}$ on a Hilbert manifold $M$ and denote by $I$ the interval $[0,1]$. Then all horizontal curves in $M$ can be parametrized on $I$. Let $\Omega_{x_{0}}(I, \mathcal{F})$ be the space of horizontal curves (i.e. tangent to $\mathcal{F}$ and of class $H^{1}$ ) with fixed 
origin $x_{0}$. In general, without more hypotheses we cannot define a Hilbert structure on $\Omega_{x_{0}}(I, \mathcal{F})$ if we associate to each horizontal curve its control as in finite dimension. So if we denote by End : $\Omega_{x_{0}}(I, \mathcal{F}) \rightarrow G$ the map which associates to each curve $\gamma$ its end point $\gamma(1)$, we cannot define the abnormal curves as "the singular points" of the map End as we can do in finite dimension. However we can still define an abnormal curve as a curve $\gamma$ which can lift to a non-trivial curve $\Gamma$ in the annulator $\mathcal{F}^{\perp}$ and which is tangent to the kernel of the 2-closed form induced on the manifold $\mathcal{F}^{\perp}$ in $T^{*} M$ by the canonical symplectic form of $T^{*} M$. Each horizontal curve which is not abnormal is called normal.

Consider a left-invariant distribution $\mathcal{F}$ on a 2-step nilpotent Hilbert Lie group $G$. Then we can show that a curve $\gamma$ is abnormal if and only if there exists a lift $\Gamma$ in $\mathcal{F}^{\perp}$ which is constant almost everywhere and moreover (as in finite dimension) we also have

THEOREM 4.1 (cf. [Be]). Let $G$ be a 2-step nilpotent Hilbert-Schmidt Lie group and $\mathcal{F}$ the left-invariant distribution generated by $F$. The space of horizontal curves $\Omega_{x_{0}}(I, \mathcal{F})$ of class $H^{1}$ is diffeomorphic to $L^{2}(I, F)$. Every horizontal curve $\gamma$ is abnormal if and only if $\gamma$ is a singular point of the map End.

The purpose of this section is to give, in the context of 2-step nilpotent Hilbert Lie groups, some "localization" of abnormal curves in terms of existence of submanifolds in which such a curve is normal for the induced distribution.

4.2. Characteristic manifolds. Let $G$ be a 2-step nilpotent Hilbert Lie group which is connected and simply connected. Denote by $\mathcal{G}=F \oplus \mathcal{Z}$ its Lie algebra and by $\mathcal{F}$ the left-invariant distribution defined by $F$ on $G$. Let $R: \mathcal{G} \rightarrow \mathcal{G}^{*}$ be the Riesz representation. Given any horizontal curve $\gamma$, the abnormality set of $\gamma$ in $G$, denoted by $\mathcal{A}_{\gamma}$, is the set of $\lambda \in \mathcal{G}^{*}$ such that $\lambda(u)=0$ for all $u \in F$ and $\lambda\left(\left[d L_{\gamma(t)}^{-1} \dot{\gamma}(t), v\right]\right)=0$ for all $v \in \mathcal{G}$. In fact, for a non-zero $\lambda \in \mathcal{A}_{\gamma}$, the curve $t \mapsto \Gamma(t)=\left(\left(d L_{\gamma(t)}^{-1}\right)^{*} \lambda, \gamma(t)\right)$ is a lift of $\gamma$ in the annulator $\mathcal{F}^{\perp}$ of the left-invariant distribution on $G$ defined by $F$ and it is tangent to the kernel of the closed 2 -form induced on the manifold $\mathcal{F}^{\perp}$ by the canonical symplectic form of the cotangent bundle of $G$ (see $[\mathrm{Be}]$ ). So, a curve is abnormal if and only if $\mathcal{A}_{\gamma}$ is non-zero.

Definition 4.1 (cf. [Mo]). A connected submanifold $S$ of $G$ is called a characteristic manifold for an abnormal curve $\gamma:[0,1] \rightarrow G$ iff:

i) $T S \cap \mathcal{F}$ have a constant dimension,

ii) $\gamma$ is tangent almost everywhere to $T S \cap \mathcal{F}$,

iii) $\gamma$ is normal in $S$ (relatively to $T S \cap \mathcal{F}$ ).

Let $\gamma:[0,1] \rightarrow G$ be an abnormal curve and $g=\gamma(0)$. Then $\widetilde{\gamma}=L_{g}^{-1}(\gamma)$ is an abnormal curve defined on $I$ and such that $\widetilde{\gamma}(0)=e$. A manifold $S$ is a characteristic manifold for $\gamma$ if and only if $\widetilde{S}=L_{g}^{-1}(S)$ is a characteristic manifold for $\widetilde{\gamma}$. So it is sufficient to study the existence of characteristic manifolds for abnormal curves $\gamma:[0,1] \rightarrow G$ such that $\gamma(0)=e$.

Let $\gamma:[0,1] \rightarrow G$ be an abnormal curve such that $\gamma(0)=e$. We denote by $\mathcal{P}$ the set of closed subspaces $K$ of $F$ such that $\gamma$ is tangent to the left-invariant distribution 
generated by $K$. It is easy to see that if $K$ belongs to $\mathcal{P}$ then the set $\left\{d L_{\gamma(t)}^{-1} \dot{\gamma}(t)\right\}$ is included in $K$. On the other hand $\mathcal{P}$ is an inductive set and from Zorn's lemma, it has a minimal element $H$. Denote by $\mathcal{H}$ the Lie subalgebra generated by $H$, that is

$$
\mathcal{H}=H \oplus[H, H] .
$$

Let $G_{H}$ be the connected and simply connected Lie subgroup of $G$ whose Lie algebra is $\mathcal{H}$. Then we have the following theorem.

THEOREM 4.2.

1) $\mathcal{P}$ has a (unique) smallest element $H$ which is the closure of the vector space generated by $\left\{d L_{\gamma(t)}^{-1} \dot{\gamma}(t)\right\}$.

2) $G_{H}$ is a characteristic manifold for $\gamma$.

3) If $G^{\prime}$ is another connected and simply connected Lie group which is also a characteristic manifold for $\gamma$ then $G^{\prime}=G_{H}$.

Proof.

1) Let $H$ be the closed vector space of $\mathcal{G}$ generated by $\left\{d L_{\gamma(t)}^{-1} \dot{\gamma}(t)\right\}$ and $\mathcal{H}=H \oplus[H, H]$ the Lie algebra generated by $H$. By the definition of $H, \gamma$ is tangent to the left-invariant distribution generated by $H$, so $H \in \mathcal{P}$. Moreover if $L \in \mathcal{P}$ then $L$ contains $H$, so $H$ is the smallest element of $\mathcal{P}$ and it is unique.

2) Suppose that $\gamma$ is abnormal in $G_{H}$ and denote by $\mathcal{A}_{\gamma}$ its abnormality set. Consider the set $\mathcal{K}$ of all $u \in \mathcal{H}$ such that for every $\lambda \in \mathcal{A}_{\gamma}$ we have $\lambda(u)=0$ and $\lambda([u, v])=0$ for all $v \in \mathcal{H}$. It is easy to see that $\mathcal{K}$ is a Lie sub-algebra of $\mathcal{H}$ which is strictly included in $\mathcal{H}$.

If we set

$$
H^{\prime}=\mathcal{K} \cap H,
$$

the Lie algebra generated by $H^{\prime}$ is

$$
H^{\prime} \oplus\left[H^{\prime}, H^{\prime}\right] \varsubsetneqq \mathcal{K} .
$$

As $H^{\prime} \subset \mathcal{K}, \gamma$ is tangent to the left-invariant distribution generated by $H^{\prime}$. On the other hand, $H^{\prime} \varsubsetneqq H$ which implies that $H$ is not minimal and gives rise to a contradiction.

3) Let $G^{\prime}$ be a connected and simply connected subgroup of $G$ which is also a characteristic manifold for $\gamma$. The subspace $T_{e} G^{\prime} \cap F$ belongs to $\mathcal{P}$, so it contains $H$. Hence, the Lie algebra $\mathcal{G}^{\prime}$ of $G^{\prime}$ contains $\mathcal{H}$, so $G^{\prime}$ contains $G_{H}$. Suppose $G^{\prime} \neq G_{H}$ as we have connected sets then $\mathcal{G}^{\prime} \supset \mathcal{H}$ and $\mathcal{G}^{\prime} \neq \mathcal{H}$ which implies that there exists a non-zero $\lambda \in \mathcal{H}^{\perp} \subset \mathcal{G}^{*}$ and for such a $\lambda$ we have then $\lambda(u)=0$ for every $u$ of $\mathcal{H}$; in particular

$$
\lambda\left(d L_{\gamma(t)}^{-1} \dot{\gamma}(t)\right)=0 \text { and } \lambda\left(\left[d L_{\gamma(t)}^{-1} \dot{\gamma}(t), v\right]\right)=0 \text { for all } v \text { of } \mathcal{H},
$$

so $\lambda$ belongs to the abnormality set of $\gamma$ in $G_{H}$, that is $\gamma$ is abnormal in $G_{H}$, and we have a contradiction.

COROLLARY 4.1. Let $G$ be a 2-step nilpotent Hilbert Lie group which is connected and simply connected, $\mathcal{G}=F \oplus \mathcal{Z}$ its Lie algebra, $\mathcal{F}$ the left-invariant distribution defined by $F$ and $\gamma$ a horizontal curve with origin e. There exists a unique connected and simply connected subgroup $G_{\gamma}$ in which $\gamma$ is normal relatively to the left-invariant distribution 
induced by $\left(G_{\gamma}, \mathcal{F} \cap T G_{\gamma}\right)$. The Lie algebra of $G_{\gamma}$ is generated by $\left\{d L_{\gamma(t)}^{-1} \dot{\gamma}(t): t \in[0,1]\right\}$. In particular, if $\overline{\operatorname{Im} \Lambda} \neq \mathcal{Z}$ then all horizontal curves are abnormal.

4.3. Application. Classical examples of 2-step nilpotent Lie algebras are Heisenberg algebras which play an important role in mathematical physics. Below we define three types of Heisenberg algebras.

4.3.1. Classical Heisenberg Lie algebras. Let $K$ be a separable Hilbert space and $K^{*}$ its dual. We define a Lie algebra structure on the space

$$
\mathcal{G}=F \oplus Z
$$

with $F=K^{*} \oplus K$ and $Z=\mathbb{R}$ as follows:

If $\left\{X_{\alpha}: \alpha \in \mathbb{N}\right\}$ is a Hilbert basis of $K$ in $F$ and $\left\{X_{\beta}^{*}: \beta \in \mathbb{N}\right\}$ is the dual basis, then we set

$$
\left[X_{\alpha}, X_{\beta}^{*}\right]=C_{\alpha \beta} \delta_{\alpha \beta} .1
$$

where $C_{\alpha \beta}$ are constants; the other brackets are zero modulo permutations. When $\sum_{\alpha, \beta}\left(C_{\alpha \beta}\right)^{2}<\infty$ the Lie algebra is of Hilbert-Schmidt type.

If $C_{\alpha \beta}=1$ for every $\alpha, \beta$, we get the classical Heisenberg algebra.

4.3.2. Generalized Heisenberg algebras. Let $K$ and $H$ be two separable Hilbert spaces. It is well known (see for example [Gu]) that if $\left\{k_{i}\right\}$ is a Hilbert basis of $K$ and $\left\{h_{\alpha}\right\}$ is a Hilbert basis of $H$ then the set of tensor products $\left\{h_{\alpha} \otimes k_{i}\right\}$ is a Hilbert basis of $\mathcal{L}_{H . S}(K ; H)$.

On $\mathcal{G}=\mathcal{L}_{H . S}(K ; H) \oplus K \oplus H$ we define a generalized Heisenberg Lie algebra structure on $\mathcal{G}=F \oplus Z$ by setting $F=\mathcal{L}_{H . S}(K ; H) \oplus K$ and $Z=H$ with Lie brackets defined, with respect to the basis $Y_{\alpha i}=\left(h_{\alpha} \otimes k_{i}, 0\right)$ and $X_{i}=\left(0, k_{i}\right)$ of $F$, by

$$
\left[Y_{\alpha i}, X_{i}\right]=C_{\alpha i} z_{\alpha}
$$

where $C_{\alpha i}$ are constants; the other brackets which are not the opposite are zero.

This Lie algebra is of Hilbert-Schmidt type if $\sum_{i, \alpha}\left(C_{i \alpha}\right)^{2}<\infty$.

If $\operatorname{dim} H=1$ then we obtain the classical Heisenberg algebras.

4.3.3. Lie Algebras of type $(\mathrm{HG})$. Let $(F, Z, \Lambda)$ be a 2 -step nilpotent Hilbert Lie algebra. Denote by $\mathcal{L}_{A . S}(F)$ the set of linear skew-symmetric continuous endomorphisms of $F$. There exists a unique linear map

$$
J: Z \rightarrow \mathcal{L}_{A . S}(F)
$$

such that

$$
\left\langle J_{z}(u), v\right\rangle_{F}=\langle\Lambda(u, v), z\rangle_{Z} \quad \forall u, v \in F \text { and } \forall z \in Z .
$$

Following Eberlein and Kaplan's work on generalized Heisenberg algebras in finite dimension ([Eb], [Ka1] and [Ka2]) we can define Lie algebras of generalized Heisenberg (HG) type as follows: 
Definition 4.2. We say that the Lie algebra associated to $(F, Z, J)$ is of type $(\mathrm{HG})$ iff:

1) $I=\bigcap_{z \neq 0} \overline{\operatorname{Im} J_{z}} \neq\{0\}$,

2) $\left\|J_{z}(u)\right\|=\|z\|\|u\| \quad \forall z \in Z$ and $\forall u \in I$.

If $I=F$ we say that $(F, Z, J)$ is non-degenerate.

Proposition 4.1. All generalized Heisenberg algebras (see Subsection 4.3.2) are of type (HG).

Proof. By using relation (2) the map $J: Z \rightarrow \mathcal{L}_{A . S}(F)$ will be defined by

$$
\begin{aligned}
J_{z_{\alpha}}\left(Y_{\beta i}\right) & =X_{i} \quad \text { iff } \alpha \neq \beta, \\
J_{z_{\alpha}}\left(Y_{\alpha i}\right) & =0, \\
J_{z_{\alpha}}\left(X_{i}\right) & =-Y_{\alpha i} .
\end{aligned}
$$

Let $z$ be any non-zero element of $Z$ such that $z=\sum_{\alpha} \lambda_{\alpha} z_{\alpha}$.

If we set $\mathbb{N}_{Z}=\left\{\alpha: l_{\alpha} \neq 0\right\}$ then

$$
\operatorname{Im} J_{z}=\operatorname{Span}\left\{Y_{\alpha i}, X_{i}: \alpha \in \mathbb{N}_{Z}, i \in \mathbb{N}\right\},
$$

so

$$
I=\bigcap_{z \neq 0} \overline{\operatorname{Im} J_{z}}=\operatorname{Span}\left\{X_{i}: i \in \mathbb{N}\right\}=K .
$$

On the other hand, for every $u \in K$ and every $z \in Z$, we can write $u=\sum_{i} u_{i} X_{i}$, $z=\sum_{\alpha} \lambda_{\alpha} z_{\alpha}$

$$
J_{z}(u)=\sum_{\alpha \in \mathbb{N}} \lambda_{\alpha} J_{z_{\alpha}}(u)=\sum_{\alpha \in \mathbb{N}, i \in \mathbb{N}} \lambda_{\alpha} u_{i} J_{z_{\alpha}}\left(X_{i}\right)=-\sum_{\alpha \in \mathbb{N}, i \in \mathbb{N}} \lambda_{\alpha} u_{i} Y_{\alpha i},
$$

and

$$
\left\|J_{z}(u)\right\|^{2}=\sum_{\alpha \in \mathbb{N}, i \in \mathbb{N}}\left|\lambda_{\alpha}\right|^{2}\left|u_{i}\right|^{2}=\left(\sum_{\alpha \in \mathbb{N}}\left|\lambda_{\alpha}\right|^{2}\right)\left(\sum_{i}\left|u_{i}\right|^{2}\right)=\|z\|^{2}\|u\|^{2}
$$

Such a construction will be given in Subsection 4.3.4.

TheOrem 4.3. Let $(F, Z, J)$ be a Lie algebra of type (HG). Let $G$ be a connected and simply connected group associated to this Lie algebra, every abnormal curve is tangent to the left-invariant distribution generated by $I^{\perp}$, the orthogonal complement of $I$ in $F$. Moreover, if $I=\operatorname{Im} J_{z}$ for all $z \neq 0$ in $Z$, then the converse is true.

In particular if $(F, Z, J)$ is non-degenerate there are no abnormal curves except constant curves.

Proof. First if $R: \mathcal{G} \rightarrow \mathcal{G}^{*}$ is the Riesz representation then

$$
R(Z)=F^{\perp}
$$

and

$$
\left\langle J_{z}(u), v\right\rangle=\langle R(z),[u, v]\rangle \quad \forall u, v \in F \text { and } \forall z \in Z \text {. }
$$


It is easy to see that an element $u$ belongs to Ker $J_{z}$ if and only if

$$
\left\langle J_{z}(u), v\right\rangle=0 \quad \forall v \in F,
$$

which is also equivalent to

$$
\langle R(z),[u, v]\rangle=0 \quad \forall v \in F .
$$

Let $G$ be a connected and simply connected group associated to the Lie algebra $\mathcal{G}$ and let $\gamma:[0,1] \rightarrow G$ be an abnormal curve. An element $R(z)$ of $\mathcal{G}$ belongs to the abnormality set $\mathcal{A}_{\gamma}$ of $\gamma$ in $G$ if and only if

$$
R\left(\left[d L_{\gamma(t)}^{-1} \dot{\gamma}(t), \cdot\right]\right)=0
$$

which, according to the previous argument, is equivalent to

$$
d L_{\gamma(t)}^{-1} \dot{\gamma}(t) \in \operatorname{Ker} J_{z} .
$$

If $(F, Z, J)$ is a Lie algebra of type $(H G)$ then, from property 2$)$ of Definition 4.2, $J_{z}(u)$ is non-zero for all $u$ and all $z \neq 0$.

As $I=\bigcap_{z \neq 0} \overline{\operatorname{Im} J_{z}}$ and $J_{z}$ is skew-symmetric, we have

$$
\operatorname{Ker} J_{z} \subset I^{\perp} \text {. }
$$

So finally, if $\gamma$ is an abnormal curve then

$$
d L_{\gamma(t)}^{-1} \dot{\gamma}(t) \in I^{\perp}
$$

which is equivalent to

$$
\dot{\gamma}(t) \in \widetilde{I^{\perp}} \gamma(t),
$$

where $\widetilde{I^{\perp}} \gamma(t)$ is the left-invariant distribution generated by $I^{\perp}$. In general the converse is not true.

If $I=\operatorname{Im} J_{z}$ for all non-zero $z$ then it is easy to see that if a curve is tangent almost everywhere to $\widetilde{I}_{\gamma(t)}$ this curve must be abnormal.

If $(F, Z, J)$ is non-degenerate, that is if $I=F$, then $I^{\perp}=\{0\}$ and all non-constant curves are normal.

The following result is a consequence of Theorem 4.3.

Proposition 4.2. For all generalized Heisenberg Lie algebras, the abnormal curves are tangent to the left-invariant distributions generated by $\mathcal{L}_{H . S}(K ; H)$.

\subsubsection{Construction of Lie algebras of type (HG)}

Proposition 4.3. For any Hilbert spaces $F$ and $Z$ such that the dimension of $F$ is finite and the dimension of $Z$ is finite or infinite, there exists a map $J: Z \rightarrow \mathcal{L}_{\text {A.S }}(F)$ such that the Lie algebra defined by $(F, Z, J)$ is of type (HG).

Proof. Let $\left\{e_{i}: i \in \mathbb{N}\right\}$ be a Hilbert basis of $F$ and $\left\{z_{\alpha}: \alpha \in \mathbb{N}\right\}$ a Hilbert basis of $Z$. Denote by $K$ the Hilbert space generated by

$$
\left\{X_{j}=e_{2 j+1}: j \in \mathbb{N}\right\}
$$


and by $\mathbb{N}_{Z}$ the set of indices $\alpha$ of the basis of $Z$. As $\mathbb{N}_{Z} \times \mathbb{N}$ is a countable set there exists a bijection

$$
\begin{gathered}
\theta: \mathbb{N}_{Z} \times \mathbb{N} \rightarrow \mathbb{N} \\
(\alpha, i) \mapsto \theta(\alpha, i)
\end{gathered}
$$

and if $Y_{\alpha i}=e_{2 \theta(\alpha, i)}$, the Hilbert space generated by $Y_{\alpha i}$ is isometric to the space $\mathcal{L}_{H . S}(K ; Z)$.

So we can construct a generalized Heisenberg algebra and from Proposition 4.1 there exists a map $J: Z \rightarrow \mathcal{L}_{A . S}(F)$ defined in this case by

$$
\begin{aligned}
J_{z_{\alpha}}\left(e_{2 \theta(\beta, i)}\right) & =e_{2 i+1} \quad \text { if } \alpha \neq \beta \\
J_{z_{\alpha}}\left(e_{2 \theta(\alpha, i)}\right) & =0 \\
J_{z_{\alpha}}\left(e_{2 i+1}\right) & =-e_{2 \theta(\alpha, i)}
\end{aligned}
$$

and such that the Lie algebra associated to $(F, Z, J)$ is of type $(\mathrm{HG})$.

In what follows we will need the next lemma:

LEMMA 4.1 (cf. [Ka1]). Let $Z$ be a vector space of finite dimension $m$ and let $p$ be the smallest integer such that $m<8 p+2^{q}$ with $0 \leq q \leq 3$. Then there exists a map

$$
J: Z \rightarrow \mathcal{L}_{A . S}\left(\mathbb{R}^{N}\right)
$$

with $N=2^{4 p+q}$ which satisfies

$$
\left\|J_{z}(u)\right\|=\|u\|\|z\| \text {. }
$$

Proposition 4.4. Let $F$ be an infinite-dimensional Hilbert space and $Z$ a finitedimensional Hilbert space. For any infinite-dimensional subspace $I$ of $F$ there exists a map $J: Z \rightarrow \mathcal{L}_{A . S}(F)$ such that $(F, Z, J)$ is of type $(\mathrm{HG})$ with $\operatorname{Im} J_{z}=I$ for all non-zero $z$ in $Z$.

P r o of. Let $I$ be an infinite-dimensional subspace of $F, p$ be the integer defined by the relation $m<8 p+2^{q}$ with $0 \leq q \leq 3$, where $m$ is the dimension of $Z$, and set $N=2^{4 p+q}$. If $\left\{e_{i}: i \in \mathbb{N}\right\}$ is a Hilbert basis of $I$, then there exists a bijection

$$
\begin{gathered}
\theta:[1, N] \times \mathbb{N} \rightarrow \mathbb{N} \\
(\alpha, i) \mapsto \theta(\alpha, i) .
\end{gathered}
$$

Set

$$
Y_{\alpha j}=e_{\theta(\alpha, j)}
$$

and

$$
I_{l}=\operatorname{Span}\left\{Y_{1 l}, \ldots, Y_{N l}\right\} .
$$

From Lemma 4.1 there exists a map

$$
J^{l}: Z \rightarrow \mathcal{L}_{A . S}\left(I_{l}\right) \text { for all } l
$$

such that

$$
\left\|J_{z}^{l}(v)\right\|=\|v\|\|z\| \quad \forall v \in I_{l} \text { and } \forall z \in Z \text {. }
$$


Let $u \in I$ and $u_{l}$ be the orthogonal projection of $u$ on $I_{l}$. If we set

$$
J_{z}(u)=\sum_{l \in \mathbb{N}} J_{z}^{l}\left(u_{l}\right),
$$

the map $J$ is well defined because

$$
\begin{aligned}
\left\|J_{z}(u)\right\|^{2} & =\lim _{l \rightarrow \infty} \sum_{h=0}^{l}\left\|J_{z}^{l}\left(u_{l}\right)\right\|^{2}=\lim _{l \rightarrow \infty} \sum_{h=0}^{l}\left\|u_{l}\right\|^{2}\|z\|^{2} \\
& =\|z\|^{2} \lim _{l \rightarrow \infty} \sum_{h=0}^{l}\left\|u_{l}\right\|^{2}=\|z\|^{2}\|u\|^{2}
\end{aligned}
$$

and the end of the proof is then a direct consequence of Lemma 4.1.

\section{References}

[AS] A. Agrachev, A. Sarychev, Abnormal sub-Riemannian geodesics: Morse index and rigidity, Ann. Inst. H. Poincaré Anal. Non Linéaire 13 (1996), 635-690.

[AOP] M. Alcheikh, P. Orro, F. Pelletier, Singularité de l'application extrémité pour les chemins horizontaux en géométrie sous-riemannienne, Prépublication 95-09a LAMA, Université de Savoie, 1995; to be published in: Actes du colloque "Géométrie sous riemannienne et singularités".

[AP] M. Arnaudon, S. Paycha, Stochastic tools on Hilbert manifolds: interplay with geometry and physics, Comm. Math. Phys. 187 (1997), 243-260.

[BG] V. Baranovsky, V. Ginzburg, Conjugacy classes in loop groups and G-bundles on elliptic curves, Internat. Math. Res. Notices 1996, no. 15, 733-751.

[Bel] A. Bellaiche, Propriétés extrémales des géodésiques, Astérisque 84-85 (1981), 83-130.

[Be] N. Bensalem, Localisation des courbes anormales et problème d'accessibilité sur un groupe de Lie hilbertien nilpotent de degré 2, Thèse de doctorat, Université de Savoie, 1998.

[BP] N. Bensalem, F. Pelletier, Approximation des extrémales pour un système bilinéaire en dimension infinie et applications, Preprint LAMA, Université de Savoie, 1998.

[Bo] N. Bourbaki, Groupes et algèbres de Lie, Chapitres 1, 2 et 3, Hermann, Paris, 1971-72.

[Eb] P. Eberlein, Geometry of 2-step nilpotent groups with a left invariant metric, Ann. Sci. École Norm. Sup. (4) 27 (1994), 611-660.

[Ek] I. Ekeland, On the variational principle, J. Math. Anal. Appl. 47 (1974), 324-353.

[GXB] J. P. Gauthier, C. Z. Xu, A. Bounabat, An observer for infinite-dimensional skewadjoint bilinear systems, J. Math. Systems Estim. Control 5 (1995), 119-122.

[GXL] J. P. Gauthier, C. Z. Xu, P. Ligarius, An observer for infinite-dimensional dissipative bilinear systems, Comput. Math. Appl. 29 no. 7 (1995), 13-21.

[Ge] Z. Ge, Horizontal path spaces and Carnot-Carathéodory metrics, Pacific J. Math. 161 (1993), 255-286.

[Gr] M. Gromov, Carnot-Carathéodory spaces seen from within, in: Sub-Riemannian Geometry, A. Bellaïche and J.-J. Risler (eds.), Progr. Math. 144, Birkhäuser, Boston, 1996, $79-323$.

[Gu] A. Guichardet, Intégration, analyse hilbertienne, Ellipses, Paris, 1989.

[Ka1] A. Kaplan, Fundamental solutions for a class of hypoelliptic PDE generated by composition of quadratic forms, Trans. Amer. Math. Soc. 258 (1980), 147-153. 
[Ka2] A. Kaplan, Riemannian nilmanifolds attached to Clifford modules, Geom. Dedicata 11 (1981), 127-136.

[Ko] H. Konno, Geometry of loop groups and Wess-Zumino-Witten models, in: Symplectic Geometry and Quantization, Y. Maeda et al. (eds.), Contemp. Math. 179, Amer. Math. Soc., Providence, 1994, 139-160.

[La] S. Lang, Differential Manifolds, Addison-Wesley, Reading, 1972.

[LS] W. Liu, H. J. Sussmann, Shortest paths for sub-Riemannian metrics on rank-two distributions, Mem. Amer. Math. Soc. 118 (1995), no. 564.

[Mo] R. Montgomery, A survey of singular curves in sub-Riemannian geometry, J. Dynam. Control Systems 1 (1995), 49-90.

[MP] P. Mormul, F. Pelletier, Contrôlabilité complète par des courbes anormales par morceaux d'une distribution de rang 3 générique sur des variétés connexes de dimension 5 et 6, Bull. Polish Acad. Sci. Math. 45 (1997), 399-418.

[OP] P. Orro, F. Pelletier, Propriétés géométriques de quelques distributions régulières, Prépublication 97, LAMA, Université de Savoie, 1997.

[PV1] F. Pelletier, L. Valère Bouche, Le problème des géodésiques en géométrie sousriemannienne singulière, C. R. Acad. Sci. Paris Sér. I Math. 317 (1993), 71-76.

[PV2] F. Pelletier, L. Valère Bouche, Abnormality of trajectory in sub-Riemannian structure, in: Geometry in Nonlinear Control and Differential Inclusions, Banach Center Publ. 32, Warsaw, 1995, 301-317.

[PS] A. Pressley, G. Segal, Loop Groups, The Clarendon Press, Oxford Univ. Press, Oxford, 1986; Russian transl.: Mir, Moscow, 1990.

[X] C. Z. Xu, Exact observability and exponential stability of infinite-dimensional bilinear systems, Math. Control Signals Systems 9 (1996), 73-93.

[Z] M. Zhitomirskii, Rigid and abnormal line subdistributions of 2-distributions, J. Dynam. Control Systems 1 (1995), 253-294. 